\title{
Constant term evaluation for summation of $C$-finite sequences
}

\section{Qing-Hu Hou ${ }^{1}$ and Guoce Xin ${ }^{2}$}

${ }^{1}$ Center for Combinatorics, LPMC-TJKLC, Nankai University, Tianjin 300071, PR China

${ }^{2}$ Department of Mathematics, Capital Normal University, Beijing 100084, PR China

${ }^{1}$ hou@nankai.edu.cn, ${ }^{2}$ guoce.xin@gmail.com

Abstract. Based on constant term evaluation, we present a new method to compute a closed form of the summation $\sum_{k=0}^{n-1} \prod_{j=1}^{r} F_{j}\left(a_{j} n+b_{j} k+c_{j}\right)$, where $\left\{F_{j}(k)\right\}$ are $C$-finite sequences and $a_{j}$ and $a_{j}+b_{j}$ are nonnegative integers. Our algorithm is much faster than that of Greene and Wilf.

Résumé. En s'appuyant sur l'évaluation de termes constants, nous présentons une nouvelle méthode pour calculer une forme close de la somme $\sum_{k=0}^{n-1} \prod_{j=1}^{r} F_{j}\left(a_{j} n+b_{j} k+c_{j}\right)$, où les $\left\{F_{j}(k)\right\}$ sont des suites $C$-finies, et où les $a_{j}$ et les $a_{j}+b_{j}$ sont des entiers positifs ou nuls. Notre algorithme est beaucoup plus rapide que celui de Greene et Wilf.

Keywords: $C$-finite sequences, constant term, summation, closed form

\section{Introduction}

A sequence $\{F(k)\}_{k \geq 0}$ is $C$-finite (see [Zei90]) if there exist constants $c_{1}, \ldots, c_{d}$ such that

$$
F(k)=c_{1} F(k-1)+c_{2} F(k-2)+\cdots+c_{d} F(k-d), \quad \forall k \geq d .
$$

Correspondingly, the integer $d$ is called the order of the recurrence. Greene and Wilf [GW07] provided a method to compute a closed form of the summation

$$
\sum_{k=0}^{n-1} \prod_{j=1}^{r} F_{j}\left(a_{j} n+b_{j} k+c_{j}\right)
$$

where $\left\{F_{j}(k)\right\}$ are $C$-finite sequences and $a_{j}, b_{j}$ are integers satisfying $a_{j} \geq 0$ and $a_{j}+b_{j} \geq 0$. They proved that the sum must be a linear combination of the terms

$$
\prod_{j=1}^{r} F_{j}\left(\left(a_{j}+b_{j}\right) n+i_{j}\right) \quad \text { and } \quad \phi_{i_{1}, \ldots, i_{j}}(n) \prod_{j=1}^{r} F_{j}\left(a_{j} n+i_{j}\right), \quad\left(0 \leq i_{j}<d_{j}\right)
$$

where $d_{j}$ is the order of the recurrence of $\left\{F_{j}(k)\right\}$ and $\phi_{i_{1}, \ldots, i_{r}}(n)$ is a polynomial in $n$ with given degree bound. Then the explicit formula of the sum can be computed by the method of undetermined coefficients. 
In this paper, we provide another approach which is based on MacMahon's partition analysis [Mac16] and the Omega calculations [APR01, Xin04]. We first introduce an extra variable $z$ and consider the summation

$$
S(z)=\sum_{k=0}^{n-1} z^{k} \prod_{j=1}^{r} F_{j}\left(a_{j} n+b_{j} k+c_{j}\right) .
$$

Then we rewrite $S(z)$ as the constant term (with respect to $x_{1}, \ldots, x_{r}$ ) of the Laurent series

$$
f_{1}\left(x_{1}\right) f_{2}\left(x_{2}\right) \cdots f_{r}\left(x_{r}\right) \sum_{k=0}^{n-1} z^{k} \prod_{j=1}^{r} x_{j}^{-a_{j} n-b_{j} k-c_{j}},
$$

where

$$
f_{j}\left(x_{j}\right)=\sum_{k=0}^{\infty} F_{j}(k) x_{j}^{k}
$$

is the generating function. Using partial fraction decomposition, we can derive an explicit formula for $S(z)$ in terms of $\prod_{j=1}^{r} F_{j}\left(\left(a_{j}+b_{j}\right) n+i_{j}\right)$ and $\prod_{j=1}^{r} F_{j}\left(a_{j} n+i_{j}\right)$, where $0 \leq i_{j}<d_{j}$. Finally, the substitution of $z=1$ leads to a closed form of the original summation.

\section{Basic tools by partial fraction decomposition}

Let $K$ be a field. Fix a polynomial $D(x) \in K[x]$. For any polynomial $P(x) \in K[x]$, we use $\operatorname{rem}(P(x), D(x), x)$ (or $\operatorname{rem}(P(x), D(x))$ for short) to denote the remainder of $P(x)$ when divided by $D(x)$. This notation is extended for rational function $R(x)=P(x) / Q(x)$ when $Q(x)$ is coprime to $D(x)$ :

$$
\operatorname{rem}(R(x), D(x)):=\operatorname{rem}(P(x) \beta(x), D(x)), \text { if } \alpha(x) D(x)+\beta(x) Q(x)=1 .
$$

In algebraic language, the remainder is the standard representative in the quotient ring $K[x] /\langle D(x)\rangle$.

It is convenient for us to use the following notation:

$$
\left\{\frac{P(x) / Q(x)}{D(x)}\right\}=\frac{\operatorname{rem}(P(x) / Q(x), D(x))}{D(x)} .
$$

Equivalently, if we have the following partial fraction decomposition:

$$
\frac{P(x)}{Q(x) D(x)}=p(x)+\frac{r_{1}(x)}{D(x)}+\frac{r_{2}(x)}{Q(x)},
$$

where $p(x), r_{1}(x), r_{2}(x)$ are polynomials with $\operatorname{deg} r_{1}(x)<\operatorname{deg} D(x)$, then we claim that $r_{1}(x)=$ $\operatorname{rem}(P(x) / Q(x), D(x))$ and hence

$$
\left\{\frac{P(x) / Q(x)}{D(x)}\right\}=\frac{r_{1}(x)}{D(x)}
$$

Note that we do not need $\operatorname{deg} r_{2}(x)<\operatorname{deg} Q(x)$. 
The following properties are transparent:

$$
\begin{gathered}
P_{1}(x) \equiv P_{2}(x) \quad(\bmod D(x)) \Rightarrow\left\{\frac{R(x) P_{1}(x)}{D(x)}\right\}=\left\{\frac{R(x) P_{2}(x)}{D(x)}\right\} ; \\
\alpha(x) D(x)+\beta(x) Q(x)=1 \Rightarrow\left\{\frac{P(x) / Q(x)}{D(x)}\right\}=\left\{\frac{P(x) \beta(x)}{D(x)}\right\} ; \\
\left\{\frac{a R_{1}(x)+b R_{2}(x)}{D(x)}\right\}=a\left\{\frac{R_{1}(x)}{D(x)}\right\}+b\left\{\frac{R_{2}(x)}{D(x)}\right\}, \quad \forall a, b \in K .
\end{gathered}
$$

The crucial lemma in our calculation is as follows.

Lemma 1 Let $R(x), D(x)$ be as above and assume $D(0) \neq 0$. Then for any Laurent polynomial $L(x)$ with $\operatorname{deg} L(x) \leq 0$, we have

$$
\mathrm{CT}_{x} L(x)\left\{\frac{R(x)}{D(x)}\right\}=\underset{x}{\mathrm{CT}}\left\{\frac{L(x) R(x)}{D(x)}\right\},
$$

where $\mathrm{CT}_{x} g(x)$ means to take constant term of the Laurent series $g(x)$ in $x$.

Proof: By linearity, we may assume $L(x)=x^{-k}$ for some $k \geq 0$.

Assume $r(x)=\operatorname{rem}(R(x), D(x))$. Since $D(0) \neq 0$, we have the following partial fraction decomposition:

$$
\frac{r(x)}{x^{k} D(x)}=\frac{p(x)}{x^{k}}+\frac{r_{1}(x)}{D(x)}
$$

where $\operatorname{deg} p(x)<k$ and $\operatorname{deg} r_{1}(x)<\operatorname{deg} D(x)$. Then taking constant term in $x$ gives

$$
\mathrm{CT}_{x} \frac{r(x)}{x^{k} D(x)}=\mathrm{CT}_{x} \frac{r_{1}(x)}{D(x)}=\mathrm{CT}_{x}\left\{\frac{x^{-k} r(x)}{D(x)}\right\}=\mathrm{CT}_{x}\left\{\frac{x^{-k} R(x)}{D(x)}\right\} .
$$

This is just (6) when $L(x)=x^{-k}$.

Let $\mathbb{Z}$ and $\mathbb{N}$ denote the set of integers and nonnegative integers respectively. Suppose that $\{F(k)\}_{k \in \mathbb{N}}$ is a $C$-finite sequence such that

$$
F(k)=c_{1} F(k-1)+c_{2} F(k-2)+\cdots+c_{d} F(k-d)
$$

holds for any integer $k \geq d$. Then its generating function is of the form

$$
f(x)=\sum_{k=0}^{\infty} F(k) x^{k}=\frac{p(x)}{1-c_{1} x-c_{2} x^{2}-\cdots-c_{d} x^{d}},
$$

where $p(x)$ is a polynomial in $x$ of degree less than $d$. We will say that $\{F(k)\}_{k \geq \mathbb{N}}$ is a $C$-finite sequence with generating function $p(x) / q(x)$, where $q(x)=1-c_{1} x-c_{2} x^{2}-\cdots-c_{d} x^{\bar{d}}$.

It is well-known [Sta86, Section 4.2] that we can uniquely extend the domain of $F(k)$ to $k \in \mathbb{Z}$ by requiring that $(7)$ holds for any $k \in \mathbb{Z}$. The $k$-th term of the extended sequence can be given by the following lemma. 
Lemma 2 Let $\{F(k)\}_{k \in \mathbb{N}}$ be a $C$-finite sequence with generating function $p(x) / q(x)$ and $\{F(k)\}_{k \in \mathbb{Z}}$ be its extension. Then

$$
F(k)=\mathrm{CT}_{x}\left\{\frac{x^{-k} p(x)}{q(x)}\right\}=\left.\left\{\frac{x^{-k} p(x)}{q(x)}\right\}\right|_{x=0} \quad \forall k \in \mathbb{Z} .
$$

Proof: Since $q(0)=1$, the second equality holds trivially. Let

$$
G(k)=\underset{x}{\mathrm{CT}}\left\{\frac{x^{-k} p(x)}{q(x)}\right\} .
$$

Then for $k \geq 0$, applying Lemma 1 gives

$$
G(k)=\underset{x}{\mathrm{CT}} x^{-k}\left\{\frac{p(x)}{q(x)}\right\}=\mathrm{CT}_{x} x^{-k} \frac{p(x)}{q(x)}=\left[x^{k}\right] f(x)=F(k),
$$

where $\left[x^{k}\right] f(x)$ means to take the coefficient of $x^{k}$ in $f(x)$.

Therefore, by the uniqueness of the extension, it suffices to show that $G(k)$ also satisfy the recursion (7) for all $k \in \mathbb{Z}$. We compute as follows:

$$
\begin{aligned}
G(k) & -c_{1} G(k-1)-\cdots-c_{d} G(k-d) \\
& =\mathrm{CT}_{x}\left\{\frac{x^{-k} p(x)}{q(x)}\right\}-c_{1}\left\{\frac{x^{-k+1} p(x)}{q(x)}\right\}-\cdots-c_{d}\left\{\frac{x^{-k+d} p(x)}{q(x)}\right\} \\
& =\mathrm{CT}_{x}\left\{\frac{x^{-k} p(x) q(x)}{q(x)}\right\}=0 .
\end{aligned}
$$

This completes the proof.

\section{Constant term evaluation}

Let $\left\{F_{j}(k)\right\}_{k \in \mathbb{Z}}$ be $C$-finite sequences with generating functions $f_{j}(x)=p_{j}(x) / q_{j}(x)$ for $j=1,2, \ldots, r$. We denote the degree of the denominators by $d_{j}=\operatorname{deg} q_{j}(x)$. To evaluate the sum

$$
S=\sum_{k=0}^{n-1} \prod_{j=1}^{r} F_{j}\left(a_{j} n+b_{j} k+c_{j}\right)
$$

we evaluate the more general sum $S_{r}(z)$ instead, where $S_{m}(z)$ is defined by

$$
S_{m}(z)=\sum_{k=0}^{n-1} z^{k} \prod_{j=1}^{m} F_{j}\left(a_{j} n+b_{j} k+c_{j}\right), \quad 0 \leq m \leq r .
$$


The advantage is that $S_{m}(z)$ can be evaluated recursively. Since $a_{j} \geq 0$ and $a_{j}+b_{j} \geq 0$, we have $a_{j} n+b_{j} k \geq 0$ for any $n \geq 0$ and $0 \leq k<n$. By Lemmas 1 and 2 , we have

$$
\begin{aligned}
S_{m}(z) & =\sum_{k=0}^{n-1} z^{k} \prod_{j=1}^{m-1} F_{j}\left(a_{j} n+b_{j} k+c_{j}\right) \mathrm{CT}_{x}\left\{\frac{x^{-a_{m} n-b_{m} k-c_{m}} p_{m}(x)}{q_{m}(x)}\right\} \\
& =\sum_{k=0}^{n-1} z^{k} \prod_{j=1}^{m-1} F_{j}\left(a_{j} n+b_{j} k+c_{j}\right) \mathrm{CT}_{x} x^{-a_{m} n} x^{-b_{m} k}\left\{\frac{x^{-c_{m}} p_{m}(x)}{q_{m}(x)}\right\} \\
& =\mathrm{CT}_{x} x^{-a_{m} n}\left\{\frac{x^{-c_{m}} p_{m}(x)}{q_{m}(x)}\right\} \sum_{k=0}^{n-1}\left(z x^{-b_{m}}\right)^{k} \prod_{j=1}^{m-1} F_{j}\left(a_{j} n+b_{j} k+c_{j}\right) .
\end{aligned}
$$

Therefore, we obtain the recursion

$$
S_{m}(z)=\underset{x}{\mathrm{CT}} x^{-a_{m} n}\left\{\frac{p_{m}(x) x^{-c_{m}}}{q_{m}(x)}\right\} S_{m-1}\left(z x^{-b_{m}}\right) .
$$

The initial condition is $S_{0}(z)=1+z+\cdots+z^{n-1}=\frac{1-z^{n}}{1-z}$.

Let $L_{m}$ and $L_{m}^{\prime}$ be the linear operators acting on Laurent polynomials in $x_{1}, \ldots, x_{m}$ by

$$
\begin{aligned}
L_{m}\left(\prod_{j=1}^{m} x_{j}^{\alpha_{j}}\right) & =\prod_{j=1}^{m} F_{j}\left(a_{j} n-\alpha_{j}\right), \\
L_{m}^{\prime}\left(\prod_{j=1}^{m} x_{j}^{\alpha_{j}}\right) & =\prod_{j=1}^{m} F_{j}\left(\left(a_{j}+b_{j}\right) n-\alpha_{j}\right) .
\end{aligned}
$$

Then $S_{m}(z)$ have simple rational function representations.

Theorem 3 For any $0 \leq m \leq r$, there exist a polynomial $P_{m}(z)$ with coefficients being Laurent polynomials in $x_{1}, \ldots, x_{m}$ and a non-zero polynomial $Q_{m}(z) \in K[z]$ such that

$$
S_{m}(z)=\frac{L_{m}\left(P_{m}(z)\right)-z^{n} L_{m}^{\prime}\left(P_{m}(z)\right)}{Q_{m}(z)}
$$

where $L_{m}, L_{m}^{\prime}$ are defined by 11 .

Proof: We prove the theorem by induction on $m$.

Setting $P_{0}(z)=1$ and $Q_{0}(z)=1-z$, we see that the assertion holds for $m=0$. Suppose that the assertion holds for $m-1$. We can compute $P_{m}(z)$ and $Q_{m}(z)$ as follows. For brevity, we write $R(z)=P_{m-1}(z) / Q_{m-1}(z)$.

By definition $S_{m-1}(z)$ is a polynomial in $z$ of degree less than $n$. If $b_{m} \geq 0$, then $-a_{m} n \leq 0$; If $b_{m} \leq 0$, then $-a_{m} n-b_{m}(n-1) \leq 0$. Thus $x^{-a_{m} n} S_{m-1}\left(z x^{-b_{m}}\right)$ is always a Laurant polynomial of 
degree no more than 0 . Therefore, by Lemma 1 and the recursion $(10)$, we have

$$
\begin{aligned}
S_{m}(z) & =\mathrm{CT}_{x}\left\{\frac{p_{m}(x) x^{-c_{m}}}{q_{m}(x)}\right\} x^{-a_{m} n} S_{m-1}\left(z x^{-b_{m}}\right) \\
& =\mathrm{CT}_{x}\left\{\frac{p_{m}(x) x^{-c_{m}} x^{-a_{m} n} S_{m-1}\left(z x^{-b_{m}}\right)}{q_{m}(x)}\right\} \\
& =\mathrm{CT}_{x}\left\{\frac{p_{m}(x) x^{-c_{m}} x^{-a_{m} n}\left(L_{m-1}\left(R\left(z x^{-b_{m}}\right)\right)-z^{n} x^{-b_{m} n} L_{m-1}^{\prime}\left(R\left(z x^{-b_{m}}\right)\right)\right)}{q_{m}(x)}\right\} \\
& =L_{m-1} \mathrm{CT}_{x} x^{-a_{m} n} G(x, z)-z^{n} L_{m-1}^{\prime} \mathrm{CT}_{x} x^{-\left(a_{m}+b_{m}\right) n} G(x, z),
\end{aligned}
$$

where $G(x, z)$ is given by

$$
G(x, z)=\left\{\frac{p_{m}(x) x^{-c_{m}} R\left(z x^{-b_{m}}\right)}{q_{m}(x)}\right\}
$$

Now set

$$
\frac{u(x, z)}{w(z)}=\operatorname{rem}\left(x^{d_{m}-1-c_{m}} R\left(z x^{-b_{m}}\right), q_{m}(x), x\right),
$$

where $u(x, z)$ is a polynomial in $x, z$ and $w(z)$ is a polynomial in $z$. Then

$$
\begin{aligned}
G(x, z) & =\left\{\frac{p_{m}(x) x^{-d_{m}+1} \cdot x^{d_{m}-1-c_{m}} R\left(z x^{-b_{m}}\right)}{q_{m}(x)}\right\} \\
& =\left\{\frac{p_{m}(x) x^{-d_{m}+1} u(x, z) / w(z)}{q_{m}(x)}\right\} \\
& =\frac{1}{w(z)}\left\{\frac{p_{m}(x) \cdot x^{-d_{m}+1} u(x, z)}{q_{m}(x)}\right\} .
\end{aligned}
$$

Since $x^{-d_{m}+1} u(x, z)$ is a Laurent polynomial of degree in $x$ less than or equal to 0 , we obtain

$$
\begin{aligned}
S_{m}(z) & =L_{m-1} \mathrm{CT}_{x} \frac{x^{-d_{m}+1} u(x, z)}{x^{a_{m} n}}\left\{\frac{p_{m}(x)}{q_{m}(x)}\right\}-z^{n} L_{m-1}^{\prime} \mathrm{CT}_{x} \frac{x^{-d_{m}+1} u(x, z)}{x^{\left(a_{m}+b_{m}\right) n}}\left\{\frac{p_{m}(x)}{q_{m}(x)}\right\} \\
& =L_{m-1} \mathrm{CT}_{x} \frac{x^{-d_{m}+1} u(x, z)}{x^{a_{m} n}} f_{m}(x)-z^{n} L_{m-1}^{\prime} \mathrm{CT}_{x} \frac{x^{-d_{m}+1} u(x, z)}{x^{\left(a_{m}+b_{m}\right) n}} f_{m}(x) .
\end{aligned}
$$

Now set

$$
P_{m}(z)=x_{m}^{-d_{m}+1} u\left(x_{m}, z\right), \quad Q_{m}(z)=w(z) .
$$

It is then easy to check that $S_{m}(z)$ has the desired form. This completes the induction.

Remark 1. Form the above proof we see that the degree of $x_{m}$ in $P_{m}(z)$ is between $-d_{m}+1$ and 0 . Therefore the coefficients of the numerator of $S(z)$ are linear combinations of the form

$$
\prod_{j=1}^{r} F_{j}\left(a_{j} n+i_{j}\right), \quad \prod_{j=1}^{r} F_{j}\left(\left(a_{j}+b_{j}\right) n+i_{j}\right),
$$


where $0 \leq i_{j} \leq d_{j}-1$.

Remark 2. Let $\{F(k)\}$ be a sequence with generating function $p(x) / q(x)$. We call the sequence $\{\bar{F}(k)\}$ with generating function $1 / q(x)$ its primitive sequence. It is more convenient to represent $S(z)$ in terms of the primitive sequences $\left\{\bar{F}_{j}(k)\right\}$ instead of the sequences $\left\{F_{j}(k)\right\}$ themselves. The existence of a such representation is obvious since $F_{j}(k)$ is a linear combination of $\bar{F}_{j}(k)$. In this way, the coefficients of the numerator of $S(z)$ will be linear combinations of the form

$$
\prod_{j=1}^{r} \bar{F}_{j}\left(a_{j} n-i_{j}\right), \quad \prod_{j=1}^{r} \bar{F}_{j}\left(\left(a_{j}+b_{j}\right) n-i_{j}\right),
$$

where $0 \leq i_{j} \leq d_{j}-1$. Then we can take advantage of the fact $\bar{F}_{j}\left(-i_{j}\right)=0,1 \leq i_{j} \leq d_{j}-1$ if $a_{j}=0$ or $a_{j}+b_{j}=0$. The computation is similar and in a natural way. In fact, if we define

$$
\frac{P_{m}(z)}{Q_{m}(z)}=\operatorname{rem}\left(p_{m}(x) x^{-c_{m}} \frac{P_{m-1}\left(z x^{-b_{m}}\right)}{Q_{m-1}\left(z x^{-b_{m}}\right)}, q_{m}(x), x\right),
$$

then we have

$$
S_{m}(z)=\frac{\bar{L}_{m}\left(P_{m}(z)\right)-z^{n} \bar{L}_{m}^{\prime}\left(P_{m}(z)\right)}{Q_{m}(z)}
$$

where

$$
\begin{aligned}
\bar{L}_{m}\left(\prod_{j=1}^{m} x_{j}^{\alpha_{j}}\right) & =\prod_{j=1}^{m} \bar{F}_{j}\left(a_{j} n-\alpha_{j}\right), \\
\bar{L}_{m}^{\prime}\left(\prod_{j=1}^{m} x_{j}^{\alpha_{j}}\right) & =\prod_{j=1}^{m} \bar{F}_{j}\left(\left(a_{j}+b_{j}\right) n-\alpha_{j}\right) .
\end{aligned}
$$

\section{Evaluation of $S_{r}(z)$ at $z=1$}

In this section, we consider the evaluation of $S_{r}(z)$ at $z=1$, which is equals to the sum

$$
S=\sum_{k=0}^{n-1} \prod_{j=1}^{r} F_{j}\left(a_{j} n+b_{j} k+c_{j}\right)
$$

The evaluation of $S_{r}(z)$ at $z=1$ can be obtained by the following lemma.

Lemma 4 Let $f(z)=\sum_{i} f_{i} z^{i}, g(z)=\sum_{i} g_{i} z^{i}$ and $h(z)=\sum_{i} h_{i} z^{i}$ be polynomials in $z$. Suppose that

$$
S(z)=\frac{f(z)-z^{n} g(z)}{h(z)}
$$

is a polynomial in $z$ and

$$
h(z)=\sum_{i \geq e} \tilde{h}_{i}(z-1)^{i}, \quad \tilde{h}_{e} \neq 0 .
$$

Then

$$
S(1)=\frac{1}{\tilde{h}_{e}} \sum_{i}\left(f_{i}\left(\begin{array}{l}
i \\
e
\end{array}\right)-g_{i}\left(\begin{array}{c}
n+i \\
e
\end{array}\right)\right) .
$$


Proof: By expanding $f(z)-z^{n} g(z)$ at the point $z=1$, we obtain

$$
f(z)-z^{n} g(z)=\sum_{i} f_{i}(z-1+1)^{i}-g_{i}(z-1+1)^{n+i}=\sum_{j}(z-1)^{j} A_{j},
$$

where

$$
A_{j}=\sum_{i}\left(f_{i}\left(\begin{array}{l}
i \\
j
\end{array}\right)-g_{i}\left(\begin{array}{c}
n+i \\
j
\end{array}\right)\right)
$$

Since $S(z)$ is a polynomial in $z$ and $\tilde{h}_{e} \neq 0$, we have $A_{j}=0$ for any $j<e$ and and $S(1)=A_{e} / \tilde{h}_{e}$, as desired.

Remark. Alternatively, we can write

$$
S(z)=z^{n} \frac{z^{-n} f(z)-g(z)}{h(z)}
$$

A similar argument yields

$$
S(1)=\frac{1}{\tilde{h}_{e}} \sum_{i}\left(f_{i}\left(\begin{array}{c}
i-n \\
e
\end{array}\right)-g_{i}\left(\begin{array}{l}
i \\
e
\end{array}\right)\right)
$$

The algorithm CFsum for finding a closed form of the sum (15).

Input: The generating functions $p_{j}(x) / q_{j}(x)$ of $F_{j}(k)$ and the parameters $\left(a_{j}, b_{j}, c_{j}\right)$

Output: A closed formula for $S=\sum_{k=0}^{n-1} \prod_{j=1}^{r} F_{j}\left(a_{j} n+b_{j} k+c_{j}\right)$.

1. Initially set $P(z)=1$ and $Q(z)=1-z$.

2. For $j=1,2, \ldots, r$ do

Set $R(z)=P(z) / Q(z)$.

Let

$$
\frac{u(x, z)}{w(z)}=\operatorname{rem}\left(p_{j}(x) x^{-c_{j}} R\left(z x^{-b_{j}}\right), q_{j}(x), x\right) .
$$

Set $P(z)=u\left(x_{j}, z\right)$ and $Q(z)=w(z)$.

3. Set $A=B=P$.

4. For $j=1,2, \ldots, r$ do

$$
A=\sum_{i=0}^{d_{j}-1} \bar{F}_{j}\left(a_{j} n+d_{j}-i\right)\left[x_{j}^{i}\right] A, \quad B=\sum_{i=0}^{d_{j}-1} \bar{F}_{j}\left(\left(a_{j}+b_{j}\right) n+d_{j}-i\right)\left[x_{j}^{i}\right] B,
$$

where $\left[x^{i}\right] f(x)$ denotes the coefficient of $x^{i}$ in $f(x)$ and $\left\{\bar{F}_{j}(k)\right\}$ is the primitive sequence corresponding to $\left\{F_{j}(k)\right\}$.

5. Let $e$ be the lowest degree of $z$ in $Q(z+1)$ and $h=\left[z^{e}\right] Q(z+1)$. 
6. Finally, return

$$
S=\frac{1}{h} \sum_{i}\left(\left(\begin{array}{l}
i \\
e
\end{array}\right)\left[z^{i}\right] A-\left(\begin{array}{c}
n+i \\
e
\end{array}\right)\left[z^{i}\right] B\right)
$$

Our algorithm suggested a new way to look at the degree bound for the coefficients $\phi_{i_{1}, \ldots, i_{r}}(n)$. One bound is just the multiplicity of 1 as a root of $Q_{r}(z)$. To study the $e$ described in (16), it is better to use the alternative representation of $S(z)=S_{r}(z)$ :

$$
S(z)=\underset{x_{1}, \ldots, x_{r}}{\mathrm{CT}}\left(\frac{1}{x_{1}^{a_{1}} \cdots x_{r}^{a_{r}}}\right)^{n} \frac{1-\left(\frac{z}{x_{1}^{b_{1} \cdots x_{r}^{b_{r}}}}\right)^{n}}{1-\frac{z}{x_{1}^{b_{1}} \cdots x_{r}^{b_{r}}}} \prod_{j=1}^{r}\left\{\frac{p_{j}\left(x_{j}\right) x_{j}^{-c_{j}}}{q_{j}\left(x_{j}\right)}\right\} .
$$

Suppose that $\alpha_{j}$ is a root of $q_{j}(x)$ with multiplicity $\nu_{j}\left(\alpha_{j}\right)$. By partial fraction decomposition, $S(z)$ can be written as a linear combination of the terms

$$
S(z ; \boldsymbol{\alpha}, s)=\underset{x_{1}, \ldots, x_{r}}{\mathrm{CT}}\left(\frac{1}{x_{1}^{a_{1}} \cdots x_{r}^{a_{r}}}\right)^{n} \frac{1-\left(\frac{z}{x_{1}^{b_{1}} \cdots x_{r}^{b_{r}}}\right)^{n}}{1-\frac{z}{x_{1}^{b_{1}} \cdots x_{r}^{b_{r}}}} \prod_{j=1}^{r} \frac{1}{\left(1-x_{j} / \alpha_{j}\right)^{s_{j}}},
$$

where $s_{j} \leq \nu_{j}\left(\alpha_{j}\right)$. From the discussion on Omega operator [Xin04], we see that the denominator of $S(z ; \boldsymbol{\alpha}, \boldsymbol{s})$ is given by

$$
\left(1-\frac{z}{\alpha_{1}^{b_{1}} \cdots \alpha_{r}^{b_{r}}}\right)^{s_{1}+s_{2}+\cdots+s_{r}-r+1}
$$

Therefore, by summing over all $\alpha, s$ and take common denominator, we see that

$$
e \leq \max \left\{\nu_{1}+\cdots+\nu_{r}-r+1: \alpha_{1}^{b_{1}} \cdots \alpha_{r}^{b_{r}}=1 \text { and } q_{j}\left(\alpha_{j}\right)=0\right\} .
$$

\section{Examples}

We have implement the algorithm CFSum in Maple, which can be download from http://www.combinatorics.net.cn/homepage/xin/maple/CFsum.txt .

Example 1. Let

$$
f(n)=\sum_{k=0}^{n-1} F(k)^{2} F(2 n-k)
$$

where $\{F(k)\}$ is the Fibonacci sequence defined by

$$
F(0)=0, F(1)=1, F(k)=F(k-1)+F(k-2), \forall k \geq 2 .
$$

We see that the generating function for $\{F(k)\}$ is $x /\left(1-x-x^{2}\right)$. Using the package, we immediately derive that

$$
f(n)=\frac{1}{2}\left(-\bar{F}(2 n)+\bar{F}(2 n-1)+\bar{F}(n)^{3}+\bar{F}(n) \bar{F}(n-1)^{2}-\bar{F}(n-1) \bar{F}(n)^{2}\right),
$$


where $\{\bar{F}(k)\}$ is the primitive sequence of $\{F(k)\}$. In fact, $\bar{F}(k)=F(k+1)$ and hence

$$
f(n)=\frac{1}{2}\left(-F(2 n+1)+F(2 n)+F(n+1)^{3}+F(n+1) F(n)^{2}-F(n) F(n+1)^{2}\right) .
$$

Example 2. Let

$$
S(z)=\sum_{k=0}^{n-1} F(k)^{4} z^{k}
$$

where $\{F(k)\}$ is the Fibonacci sequence defined as in Example 1. Using the package, we find that

$$
\begin{aligned}
S(z) & =\frac{\sum_{i=0}^{4} f_{i}(z) z^{n} \bar{F}(n-1)^{i} \bar{F}(n)^{4-i}-z(z+1)\left(z^{2}-5 z+1\right)}{(z-1)\left(z^{2}+3 z+1\right)\left(z^{2}-7 z+1\right)} \\
& =\frac{\sum_{i=0}^{4} f_{i}(z) z^{n} F(n)^{i} F(n+1)^{4-i}-z(z+1)\left(z^{2}-5 z+1\right)}{(z-1)\left(z^{2}+3 z+1\right)\left(z^{2}-7 z+1\right)},
\end{aligned}
$$

where

$$
f_{0}(z)=z(z+1)\left(z^{2}-5 z+1\right), f_{1}(z)=-4 z^{2}\left(z^{2}-3 z-1\right), f_{2}(z)=6 z^{2}\left(z^{2}-z+1\right),
$$

and

$$
f_{3}(z)=-4 z^{2}\left(z^{2}+3 z-1\right), \quad f_{4}(z)=z^{4}+11 z^{3}-14 z^{2}-5 z+1 .
$$

\section{D-finite sequence involved}

The readers are referred to [Sta99, Chapter 6.4] for definitions of D-finite generating functions and Precursive sequence. Let $\{G(k)\}_{k \in \mathbb{N}}$ be a P-recursive sequence with D-finite generating function $g(x)$. We wish to find a similar representation of the sum

$$
S=\sum_{k=0}^{n-1} \prod_{j=1}^{r+1} F_{j}(k)
$$

with $F_{j}$ as before except for $F_{r+1}(k)=G(k)$ being P-recursive. We shall only consider the case $c_{r}=0$ for brevity.

Define $S_{m}(z)$ as in (9). The recursion (10) still holds for $m \leq r$, and a similar calculation yields

$$
S_{r+1}(z)=\underset{x}{\mathrm{CT}} x^{-a_{r+1} n} g(x) S_{r}\left(z x^{-b_{r+1}}\right) .
$$

By Theorem 3 we can write

$$
S_{r}(z)=\frac{L_{r}\left(P_{r}(z)\right)-z^{n} \tilde{L}_{r}\left(P_{r}(z)\right)}{Q_{r}(z)}
$$


Since $S_{r}(z)$ is a polynomial in $z$ of degree less than $n, \operatorname{deg} P_{r}(z)<\operatorname{deg} Q_{r}(z)$ and $Q_{r}(0) \neq 0$.

Now put (17) into the recursion and set $z=1$, we obtain

$$
S_{r+1}(1)=\operatorname{CT}_{x} x^{-a_{r+1} n} g(x) \frac{L_{r}\left(P_{r}\left(x^{-b_{r+1}}\right)\right)-x^{-n b_{r+1}} \tilde{L}_{r}\left(P_{r}\left(x^{-b_{r+1}}\right)\right)}{Q_{r}\left(x^{-b_{r+1}}\right)}
$$

This expression can be written as

$$
S_{r+1}(1)=\mathrm{CT}_{x} x^{-a_{r+1} n} \bar{g}(x) L_{r}(\bar{P}(x))-x^{-n\left(a_{r+1}+b_{r+1}\right)} \tilde{L}_{r}(\bar{P}(x)) \bar{g}(x),
$$

where $\bar{g}(x)=g(x) \bar{Q}(x)^{-1}$, with

$$
\frac{P_{r}\left(x^{-b_{r+1}}\right)}{Q_{r}\left(x^{-b_{r+1}}\right)}=\frac{\bar{P}(x)}{\bar{Q}(x)}
$$

being in its standard representation.

Now if we let $\bar{G}(k)=\left[x^{k}\right] \bar{g}(x)$. Then we have a representation of $S_{r+1}(1)$ by a linear combination of terms of the form

$$
\prod_{j=1}^{r+1} \bar{F}_{j}\left(u_{j} n+v_{j}\right)
$$

where $\bar{F}_{j}(k)$ is the primitive sequence of $F_{j}(k)$ as before, except that $\bar{F}_{r+1}(k)=\bar{G}(k)$.

It is clear that $\bar{g}(x)$ is also D-finite and hence $\bar{G}(k)$ is P-recursive. It can be shown that if $G(k)$ satisfy a P-recursion of order $e$ then we can find for $\bar{G}(k)$ a P-recursion of order $e+\operatorname{deg} \bar{Q}(x)$.

\section{Acknowledgements}

\section{References}

[APR01] G.E. Andrews, P. Paule, and A. Riese. Macmahon's partition analysis iii: the Omega package. Europ. J. Combin., 22:887-904, 2001.

[GW07] C. Greene and H.S. Wilf. Closed form summation of C-finite sequences. Trans. AMS, 359(3):1161-1190, 2007.

[Mac16] P.A. MacMahon. Combinatory Analysis, 2 volumes. Cambridge University, 1915-1916. (Reprinted: Chelsea, New York, 1960).

[Sta86] R.P. Stanley. Enumerative Combinatorics, I. Wadsworth \& Brooks/Cole, 1986. (Reprinted: Cambridge University Press, 1997).

[Sta99] R.P. Stanley. Enumerative Combinatorics, II. Cambridge University Press, 1999.

[Xin04] G. Xin. A fast algorithm for MacMahon's partition analysis. Electron. J. Combin., 11:R58, 2004.

[Zei90] D. Zeilberger. A holonomic systems approach to special functions identities. J. Comput. Appl. Math., 32:321-368, 1990. 
